\title{
Revisión sistemática: el más alto nivel de evidencia
}

\author{
Systematic review: the highest level of evidence \\ Marlene Vanessa Salcido Reyna, *Alexis Vargas del Toro, ${ }^{*}$ \\ Natasha Alexandra Medina Vincent, ${ }^{*}$ Fernanda Ramírez Amado, ${ }^{*}$ Martín Octavio García Salazar, * \\ Andrea Melissa Briseño González, * José María Jiménez Ávila* \\ *Instituto Tecnológico y de Estudios Superiores de Monterrey, Campus Guadalajara. Guadalajara, Jalisco, México.
}

\begin{abstract}
Resumen
La revisión sistemática es una publicación de fuente secundaria, es decir, resume la información de artículos primarios; sin embargo, es necesario que se describa el proceso de elaboración de una manera transparente y comprensible, desde la recolección de artículos, los criterios de inclusión, la evaluación de los artículos y el análisis de los resultados, para que sea sistemática. Podemos hacer una revisión de un tema sin que sea sistemática, la diferencia siempre es el grado de evidencia, el metaanálisis es el análisis estadístico de dos o más estudios, no necesariamente estos artículos provienen de una revisión sistemática, ni todas las revisiones sistemáticas tienen metaanálisis. La revisión sistemática pretende contestar una pregunta de investigación científica. El proceso de elaboración lo podemos dividir en etapas sencillas; sin embargo, es importante evidenciar lo que se hizo en el estudio, así como las fechas de búsqueda: 1 . Definir la pregunta de investigación, 2. Establecer los criterios de elegibilidad, 3. Establecer la estrategia de búsqueda, 4. Analizar el sesgo y los resultados de los estudios elegidos, y 5 . Interpretación y presentación de los resultados.
\end{abstract}

Palabras clave: Revisión sistemática, metaanálisis, medicina basada en evidencia, Cochrane.

\begin{abstract}
The systematic review is a publication of secondary source, that is, it summarizes the information of primary articles, however it is necessary that the elaboration process is described in a transparent and understandable way, from the collection of articles, the criteria of the articles and the analysis of the results, to make it systematic. We can make a review of a topic without it being systematic, the difference is always the degree of evidence, the meta-analysis is the statistical analysis of two or more studies, these articles do not come from a systematic review, nor do all systematic reviews have meta-analysis. Systematic review is a scientific investigation that attempts to answer a research question. The elaboration process can be divided into simple stages, but it is important to show what was done in the study as well as the search dates. 1. Define the research question, 2. Establish eligibility criteria, 3. Establish the search strategy, 4. Analyze the bias and the results of the chosen studies, and 5. Interpretation and presentation of the results.
\end{abstract}

Keywords: Systematic review, meta-analysis, evidence-based medicine, Cochrane.

\section{Introducción}

La revisión sistemática es una investigación fundamental para la medicina basada en evidencia, ya que proveen el más alto nivel e interviene en la toma de decisiones médicas e incluso administrativas. El objetivo es el reconocimiento de la evidencia disponible, el nivel de validez de dicha información y el resumen de la misma. ${ }^{1}$

Correspondencia:

Marlene Vanessa Salcido Reyna

E-mail: drasalcidoreyna@gmail.com

Recibido: 22-08-2021. Aceptado: 23-08-2021.
Archie Cochrane dijo: «[...] ciertamente, se puede hacer una gran crítica a la profesión médica, por no haber organizado un resumen crítico por especialidad o subespecialidad, actualizado periódicamente, todos los ensayos clínicos controlados que sean relevantes...».2

En la actualidad, el acceso a la información es más sencillo a través de los medios electrónicos a bases de datos bibliográficas (Medline, PubMed,

Citar como: Salcido RMV, Vargas TA, Medina VNA, Ramírez AF, García SMO, Briseño GAM, et al. Revisión sistemática: el más alto nivel de evidencia. Orthotips. 2021; 17 (4): 217-221. https://dx.doi. org/10.35366/102220 
Tabla 1: Diferencia entre revisiones.

\begin{tabular}{lll}
\hline Revisión narrativa & Revisión sistemática & Metaanálisis \\
\hline $\begin{array}{ll}\text { No tiene metodología definida } \\
\text { Lo puede realizar una sola persona }\end{array}$ & Búsqueda exhaustiva de la literatura & Requiere de un análisis estadístico \\
No son reproducibles & Deben ser por lo menos 2 revisores & Se evalúa la heterogeneidad entre los estudios \\
Criterio implícito de un individuo & Se evalúa la calidad de los estudios incluidos & Se obtiene una media sumaria \\
\end{tabular}

Embase, LILACS, Cochrane, etcétera); sin embargo, nos enfrentamos a dos circunstancias, la primera es la gran cantidad de información; existe una explosión de información, más de dos millones de artículos son publicados al año, en más de 20,000 revistas médicas; y la segunda, no contiene toda la evidencia disponible cuando recurrimos a sólo una base de datos, por ejemplo Medline, se ha estimado que en una búsqueda electrónica sólo conseguirá identificar de 60 a $70 \%$ de los ensayos clínicos. ${ }^{2}$

Una revisión sistemática tiene como objetivo reunir toda la evidencia empírica que cumple unos criterios de elegibilidad previamente establecidos, con el fin de responder una pregunta específica de investigación. Utiliza métodos sistemáticos y explícitos, que se eligen con el fin de minimizar sesgos, aportando así resultados más fiables, a partir de los cuales se pueden extraer conclusiones y tomar decisiones. ${ }^{1}$

\section{Características de una revisión sistemática}

1. Objetivos claramente establecidos con criterios de elegibilidad de estudios previamente definidos.

2. Metodología explícita y reproducible.

3. Búsqueda sistemática que identifique todos los estudios que puedan cumplir los criterios de elegibilidad.

4. Evaluación de la validez de los resultados de los estudios incluidos.

5. Una presentación sistemática.

6. Una síntesis de las características y resultados de los estudios incluidos.

La revisión sistemática exige un método riguroso y explícito para la identificación, evaluación crítica y síntesis de la evidencia obtenida, ${ }^{2}$ mientras que en la revisión narrativa, su búsqueda puede no ser

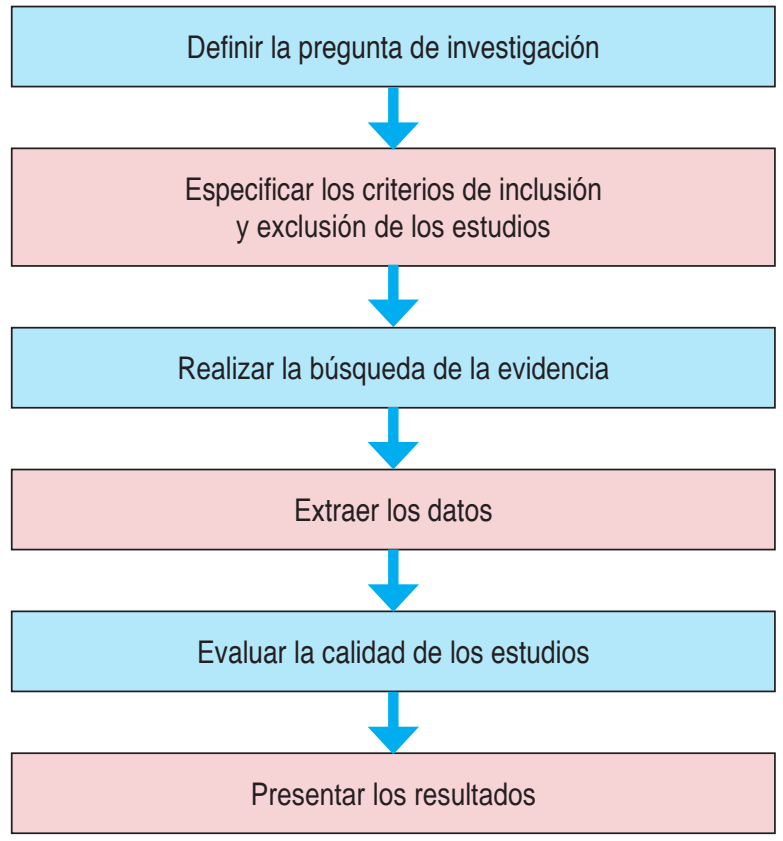

Figura 1: Pasos para realizar una revisión sistemática.

rigurosa ni necesariamente tiene que estar explícita, es decir, no se tiene que especificar la fecha de búsqueda, los buscadores utilizados y palabras clave utilizadas.

La revisión sistemática hace referencia al sistema para encontrar toda la información, evaluar y resumir los resultados; sin embargo, esta exposición puede ser de manera narrativa o cualitativa, o cuantitativa a través de un metaanálisis (el término metaanálisis fue introducido por Glass en 1976, quien lo definió como un análisis estadístico de una amplia serie de análisis de resultados de estudios individuales con el objeto de integrar su hallazgos). ${ }^{2}$ De la misma manera, para hacer un metaanálisis no es necesario realizar una búsqueda sistemática, se pueden metaanalizar varios artículos sin importar la metodología de búsqueda (Tabla 1). ${ }^{1}$ 


\section{Limitaciones de la revisión sistemática y metaanálisis}

Puede incluir menos estudios, es una publicación de fuente secundaria, es decir, depende de los estudios primarios, es un estudio observacional, retrospectivo, susceptible a sesgos. El metaanálisis es un instrumento para la generación de hipótesis y no para la toma de decisiones, es complementario no sustituto del ensayo clínico aleatorizado de gran tamaño. ${ }^{2}$

\section{Pasos para realizar una revisión sistemática (Figura 1)}

\section{Paso 1. Definir la pregunta de investigación}

La pregunta define los límites de la investigación, si es muy restringida limitará la búsqueda, identificando pocos estudios, si es muy amplia dificultará el análisis y llegar a conclusiones aplicables. ${ }^{3}$

El modelo más frecuente es definir la pregunta bajo la nemotecnia PICOT, la población se define como un grupo de personas con una condición, ${ }^{3}$ por ejemplo, se puede realizar una búsqueda en PubMed utilizando la palabra gonartrosis, se obtendrá un resultado aproximado de 42,154 artículos; sin embargo, si se desea saber qué evidencia existe en pacientes con hemofilia, se agrega este límite a la búsqueda, y se obtendrán 71 resultados, de esta manera se realiza un enfoque específico a la pregunta definida.

\section{Paso 2. Establecer los criterios de inclusión y exclusión de los estudios}

Una vez definida la pregunta de investigación y los objetivos de la búsqueda, se establecen los criterios de inclusión, que constituyen las fronteras del protocolo, es decir, tipo de estudios que se van a incluir, sus características y el tiempo, la selección de la información dependerá de los criterios de elegibilidad, ya que cada estudio se realizó de manera primaria con criterios diferentes, es fundamental que el autor defina si sólo incluye estudios en humanos, los idiomas, tipo de estudio y el tiempo. ${ }^{3}$

\begin{tabular}{|c|c|c|}
\hline Información general & Características del estudio & Resultados \\
\hline $\begin{array}{l}\text { - Quién realizó la extracción de datos } \\
\text { - Elementos del estudio } \\
\text { - Tipo de estudio } \\
\text { - País de origen } \\
\text { - Título } \\
\text { - Cita }\end{array}$ & $\begin{array}{l}\text { - Objetivos } \\
\text { - Diseño } \\
\text { - Criterios de inclusión } \\
\text { - Intervención } \\
\text { - Participantes } \\
\text { - Control }\end{array}$ & $\begin{array}{l}\text { - Variables obtenidas } \\
\text { - Pruebas realizadas } \\
\text { - Valor de p }\end{array}$ \\
\hline
\end{tabular}

Figura 2: Datos que debe incluir la hoja de extracción de datos.

\section{Generación de secuencia aleatoria (sesgo de selección) \\ Ocultación de la ubicación (sesgo de selección)}

Enceguecimiento de los participantes y del personal (sesgo de desempeño)

Ocultación de la evaluación de resultados (sesgo de detección): resultados autorreportados

Ocultación de la evaluación de resultados (sesgo de detección): mediciones objetivas

Datos de resultados incompletos (eliminación de datos): todos los resultados

Reportes selectivos (sesgo de reportes)

Otros sesgos
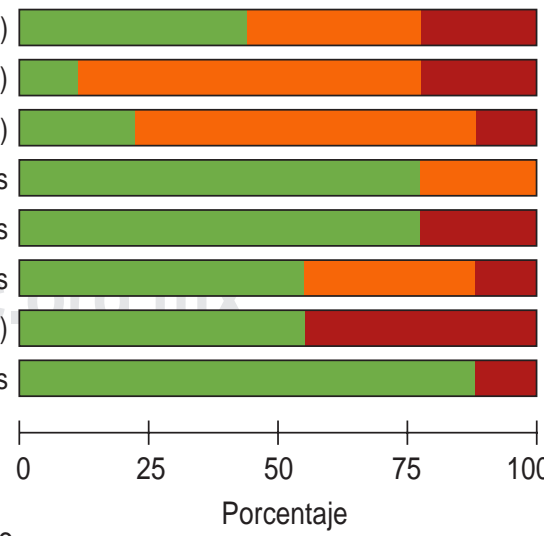

Bajo riesgo de sesgo $\square$ Riesgo de sesgo no claro $\square$ Alto riesgo de sesgo 


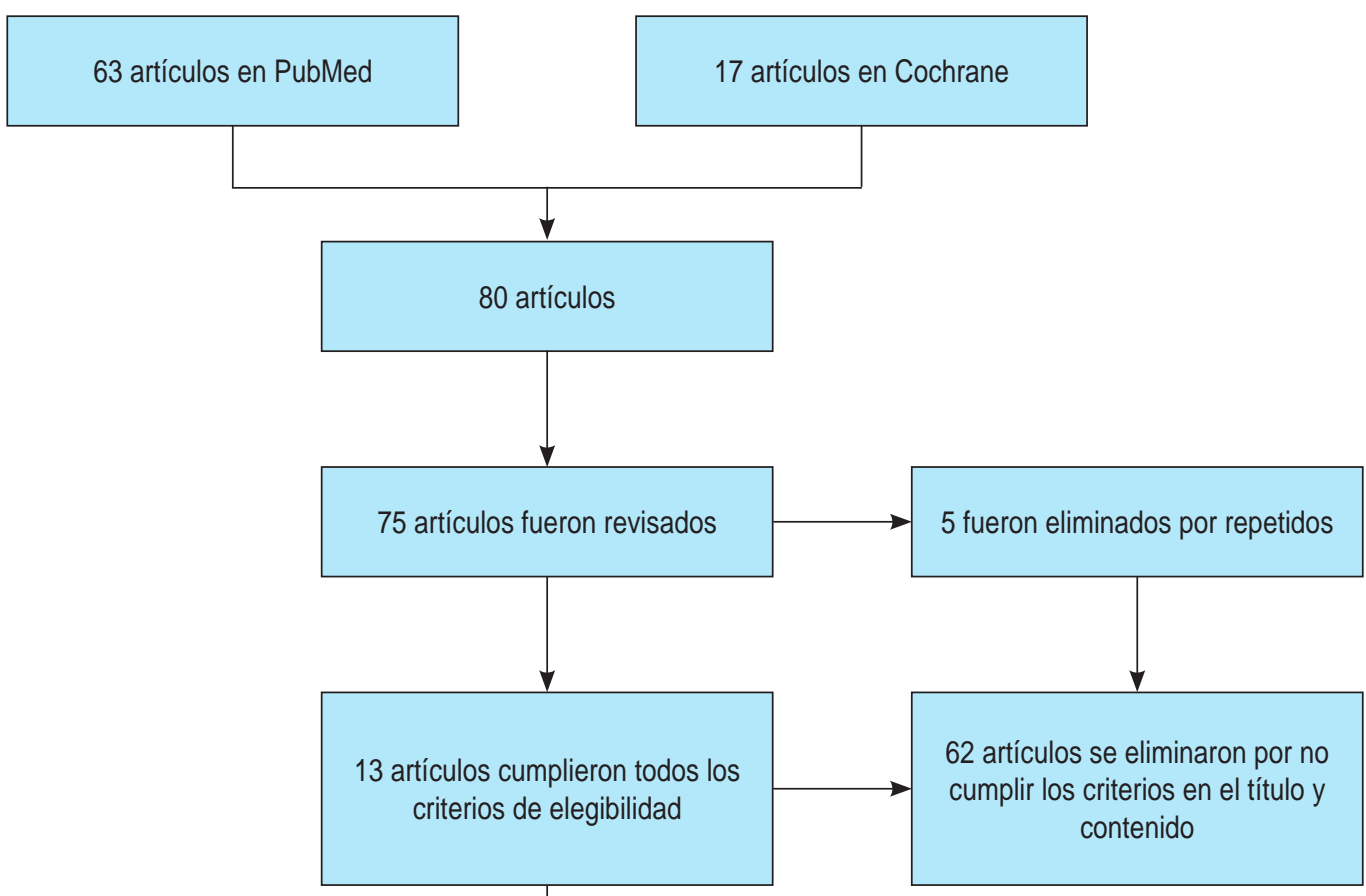

Figura 4:

13 artículos fueron incluidos en total en el estudio

\section{Paso 3. Realizar la búsqueda de la evidencia}

Debe ser explícita y se debe referir la fecha en que se realizó, así como el buscador, términos incluidos y límites en tiempo de publicación, tipos de estudios. Realizar la búsqueda en inglés y en español, Cochrane recomienda al menos utilizar tres bases de datos, las estándar son PubMed, Embase y Cochrane, ${ }^{4}$ se puede agregar Google Scholar, ya que permite incluir la búsqueda en español. Medline también está disponible mediante suscripción a través de proveedores de bases de datos como OVID o EBSCO. ${ }^{5}$ EMBASE proporciona una mejor cobertura de las revistas en Europa, India y más revistas en el campo de farmacología ${ }^{3}$ una búsqueda en Medline sola es inadecuada y dependiendo del tema sólo se identifica entre 30 y $80 \%$ de los ensayos controlados aleatorios. ${ }^{3}$

\section{Paso 4. Extraer los datos}

Depende de los resultados y el tipo de análisis que se vaya a realizar, se puede realizar un diagrama donde se refiera la base de datos y la fuente, esta misma base de datos es útil para aplicar el análisis de validez y resultado. El proceso de selección de estudios debe ser explícito para que minimice el riesgo de sesgos y errores. ${ }^{2}$ Se puede utilizar algún software de gestión de referencias como EndNote o Mendeley para recopilar, almacenar y organizar las referencias. ${ }^{3}$

Es obligatorio utilizar un formulario para extracción de datos, las tres listas de verificación de apoyo a la extracción de datos más utilizadas son las de Center for Evidence-Based Medicine (CEBM), la Cochrane Collaboration y el Critical Appraisal Skills Program (CASP). ${ }^{3}$ La extracción de datos debe ser realizada por dos revisores independientes y cualquier desacuerdo debe conciliarse. Realizar estas tablas permite analizar resultados diferentes y valorar si pueden agruparse y realizar un metaanálisis. ${ }^{3}$ La extracción de datos debe incluir tres elementos: 1) información general, quién realizó la extracción de datos y elementos del estudio (tipo de estudio, país de origen, título, cita); 2) características del estudio, objetivos, diseño, criterios de inclusión, procedimientos utilizados (alea- 
torización) participantes, intervención, descripción de la intervención y control; y 3) resultados (Figura 2).

\section{Paso 5. Evaluar la calidad de los estudios}

La lectura del artículo debe ser en forma analítica y se deben aplicar las escalas de evaluación. Se debe elegir el método que mejor se adapte al tipo de revisión que se está realizando. No es la misma escala si se revisan ensayos aleatorios o estudios de cohorte. Para ensayos clínicos no aleatorios se puede utilizar escala de Newcastle Ottawa, para los ensayos clínicos aleatorios se pueden utilizar los dominios de Cochrane (Figura 3). ${ }^{3}$ Los autores suelen establecer lista de verificación de elementos necesarios para evaluar la calidad, se pueden utilizar herramientas guía como CONSORT, RTC, STROBE para estudios observacionales. Es necesario un mínimo de dos revisores independientes para evaluar la calidad de los estudios.

\section{Paso 6. Presentar los resultados ${ }^{6}$}

La síntesis implica recopilar, combinar y resumir los resultados de los estudios individuales, puede hacerse cuantitativamente utilizando el metaanálisis, o si los resultados de los estudios no pueden compararse puede hacerse un enfoque narrativo. Es importante que la síntesis considere la solidez de la evidencia, los efectos observados, las razones de las inconsistencias, la calidad evaluada, los datos extraídos y, si es posible, el metaanálisis realizado debe expresar las conclusiones. Los investigadores deben consultar la pregunta original y si hay evidencia suficiente para responder de manera concluyente. ${ }^{4}$

Para respaldar el proceso de revisión existen pautas; PRISMA es un conjunto de elementos basados en evidencia, para informar en revisiones sistemáticas y metaanálisis. Consta de una lista de verificación que contiene 27 elementos e incluye un diagrama de flujo (Figura 4). ${ }^{3}$

\section{Conclusiones}

La revisión sistemática es un tipo de investigación con el más alto nivel de evidencia, los autores adquieren conocimiento sobre el tema y permite realizar un resumen de una búsqueda amplia, minuciosa y explícita de artículos a los que se les ha realizado una evaluación sobre su validez o riesgo de sesgo. La estrategia utilizada debe ser explícita y reproducible, tiene riesgo de sesgo al igual que los demás tipos de publicación, también posee ventajas importantes, como requerir relativamente pocos recursos para su realización.

\section{Referencias}

1. Centro Cochrane Iberoamericano, traductores. Manual Cochrane de Revisiones Sistemáticas de Intervenciones, versión 5.1.0 [actualizada en marzo de 2011] [Internet]. Barcelona: Centro Cochrane Iberoamericano; 2012. Disponible en: http://www.cochrane.es/?q=es/node/269

2. Centeno-Romero M, Chacón-Arrieta G, Vega-Aguilar JA, Gonzalez-Torres A, Leitón-Jiménez J. Revisión sistemática de literatura: aplicaciones de las comunicaciones moleculares. Revista Tecnología En Marcha. 2021; 34 (2): 147-160.

3. Donato $\mathrm{H}$, Donato $\mathrm{M}$. Stages for undertaking a systematic review. Acta Med Port. 2019; 32 (3): 227-235.

4. Ferreira González I, Urrútia G, Alonso-Coello P. Systematic reviews and meta-analysis: scientific rationale and interpretation. Rev Esp Cardiol. 2011; 64 (8): 688-696.

5. Pollock A, Berge E. How to do a systematic review. Int J Stroke. 2018; 13 (2): 138-156.

6. Aromataris E, Fernandez R, Godfrey CM, Holly C, Khalil $H$, Tungpunkom P. Summarizing systematic reviews: Methodological development, conduct and reporting of an umbrella review approach. Int J Evid Based Healthc. 2015; 13 (3): $132-140$.

\section{Conflicto de intereses}

Ninguno.

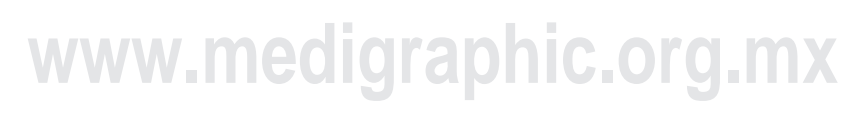

